\title{
Existence of Mild and Classical Solutions for Nonlocal Impulsive Integrodifferential Equations in Banach Spaces with Measure of Noncompactness
}

\author{
K. Karthikeyan, ${ }^{1}$ A. Anguraj, ${ }^{2}$ K. Malar, ${ }^{3}$ and Juan J. Trujillo ${ }^{4}$ \\ ${ }^{1}$ Department of Mathematics, K.S.R. College of Technology, Tiruchengode, Tamil Nadu 637215, India \\ ${ }^{2}$ Department of Mathematics, PSG College of Arts and Science, Coimbatore, Tamil Nadu 641014, India \\ ${ }^{3}$ Department of Mathematics, Erode Arts and Science College, Erode, Tamil Nadu 638 009, India \\ ${ }^{4}$ Universidad de La Laguna Departamento de Análisis Matemático, C/Astr. Fco. Snchez s/n, Tenerife, 38271 La Laguna, Spain
}

Correspondence should be addressed to K. Karthikeyan; karthi_phd2010@yahoo.co.in

Received 24 December 2013; Revised 24 May 2014; Accepted 24 May 2014; Published 19 June 2014

Academic Editor: Juan J. Nieto

Copyright (c) 2014 K. Karthikeyan et al. This is an open access article distributed under the Creative Commons Attribution License, which permits unrestricted use, distribution, and reproduction in any medium, provided the original work is properly cited.

We study the existence of mild and classical solutions are proved for a class of impulsive integrodifferential equations with nonlocal conditions in Banach spaces. The main results are obtained by using measure of noncompactness and semigroup theory. An example is presented.

\section{Introduction}

In this present paper, we are concerned with the existence of mild and classical solutions are proved for a class of impulsive integrodifferential equations with nonlocal conditions:

$$
\begin{gathered}
u^{\prime}(t)=A u(t) \\
+f\left(t, u(t), \int_{0}^{t} k(t, s, u(s)) d s, \int_{0}^{a} h(t, s, u(s)) d s\right), \\
t \in[0, a], \quad t \neq t_{i} \\
u(0)=u_{0}+g(u), \\
\Delta u\left(t_{i}\right)=I_{i}\left(u\left(t_{i}\right)\right), \quad i=1,2, \ldots, p,
\end{gathered}
$$

where $A$ is the infinitesimal generator of a $C_{0}$-semigroup $T(t)$ in a Banach space $X$ and $f:[0, a] \times X \times X \rightarrow X, k:[0, a] \times$ $[0, a] \times X \rightarrow X, h:[0, a] \times[0, a] \times X \rightarrow X, 0<t_{1}<$ $t_{2}<t_{3}<\cdots<t_{p}<a$ and $I_{i}: X \rightarrow X, i=1,2, \ldots, p$ are impulsive functions and $g: \mathrm{PC}([0, \mathrm{a}] ; \mathrm{X}) \rightarrow \mathrm{X}, \mathrm{u}_{0} \in \mathrm{X}$, and $X$ is a real Banach space with norm $\|\cdot\| . \Delta u\left(t_{i}\right)=u\left(t_{i}^{+}\right)-$ $u\left(t_{i}^{-}\right), u\left(t_{i}^{+}\right), u\left(t_{i}^{-}\right)$denote the right and left limits of $u$ at $t_{i}$, respectively.

Integrodifferential equations are important for investigating some problems raised from natural phenomena. They have been studied in many different aspects. The theory of semigroups of bounded linear operators is closely related to the solution of differential and integrodifferential equations in Banach spaces. In recent years, this theory has been applied to a large class of nonlinear differential equations in Banach spaces. We refer to the papers [1-5] and the references cited therein. Based on the method of semigroups, existence, and uniqueness of mild, strong, and classical solutions of semilinear evolution equations were discussed by Pazy [6]. In [7], Xue studied the semilinear nonlocal differential equations with measure of noncompactness in Banach spaces. Lizama and Pozo [8] investigated the existence of mild solutions for semilinear integrodifferential equation with nonlocal initial conditions by using Hausdorff measure of noncompactness via a fixed point.

In recent years, the impulsive differential equations have been an object of intensive investigation because of the wide 
possibilities for their applications in various fields of science and technology as theoretical physics, population dynamics, economics, and so forth; see [9-13]. The study of semilinear nonlocal initial problem was initiated by Byszewski $[14,15]$ and the importance of the problem lies in the fact that it more general and yields better effect than the classical initial conditions. Therefore it has been extensively studied under various conditions on the operator $A$ and the nonlinearity $f$ by several authors $[13,16-18]$.

Byszwski and Lakshmikantham [19] prove the existence and uniqueness of mild solutions and classical solutions when $f$ and $g$ satisfy Lipschitz-type conditions. Ntouyas and Tsamotas $[20,21]$ study the case of compactness conditions of $f$ and $T(t)$. Zhu et al. [22] studied the existence of mild solutions for abstract semilinear evolution equations in Banach spaces. In [23], Liu discussed the existence and uniqueness of mild and classical solutions for the impulsive semilinear differential evolution equation. In [24], the authors studied the existence of mild solutions to an impulsive differential equation with nonlocal conditions by applying DarboSadovskii's fixed point theorem. In recent paper [25], Ahmad et al. studied nonlocal problems of impulsive integrodifferential equations with measure of noncompactness. For some more recent results and details, see [26-29].

Motivated by the above-mentioned works, we derive some sufficient conditions for the solutions of integrodifferential equations (1) combining impulsive conditions and nonlocal conditions. Our results are achieved by applying the Hausdorff measure of noncompactness and fixed point theorem. In this paper, we denote by $N=\sup \{\|T(t)\|: t \in$ $[0, a]\}$. Without loss of generality, we let $u_{0}=0$.

\section{Preliminaries}

Let $(X,\|\cdot\|)$ be a real Banach space. We denote by $C([0, a] ; X)$ the space of $X$-valued continuous functions on $[0, a]$ with the norm $\|x\|=\sup \|x(t)\|, t \in[0, a]$ and by $L^{1}(0, a ; X)$ the space of $X$-valued Bochner integrable functions on $[0, a]$ with the norm $\|f\|_{L^{1}}=\int_{0}^{a}\|f(t)\| d t$.

We put $J_{i}=\left(t_{i}, t_{i+1}\right], i=1,2, \ldots, p$. In order to define the mild solution of problem (1), we introduce the following set.

$\operatorname{PC}([0, a] ; X)=\{u:[0, a] \rightarrow X: u$ is continuous on $J_{i}, i=0,1,2, \ldots, p$ and the right limit $u\left(t_{i}\right)$ exists, $i=$ $1,2, \ldots, p\}$.

Definition 1. A function $u \in \operatorname{PC}([0, a] ; X)$ is a mild solution of (1) if

$$
\begin{aligned}
u(t)= & T(t)\left[u_{0}-g(u)\right] \\
& +\int_{0}^{t} T(t-s) f\left(s, u(s), \int_{0}^{s} k(s, \tau, u(\tau)) d \tau,\right. \\
& \left.\quad \int_{0}^{a} h(s, \tau, u(\tau)) d \tau\right) d s \\
& +\sum_{0<t_{i}<t} T\left(t-t_{i}\right) I_{i}\left(u\left(t_{i}\right)\right), \quad 0 \leq t \leq a .
\end{aligned}
$$

The Hausdorff measure of noncompactness $\beta_{\mathbb{Y}}(B)$ is defined by $\beta_{\mathbb{Y}}(B)=\inf \{r>0, B$ can be covered by finite number of balls with radii $r$ for bounded set $B$ in a Banach space $Y$.

Lemma 2 (see [30]). Let $\mathbb{Y}$ be a real Banach space and $B ; C \subseteq$ $\checkmark$ be bounded, with the following properties:

(1) $B$ is precompact if and only if $\beta_{\mathbb{X}}(B)=0$;

(2) $\beta_{\mathbb{Y}}(B)=\beta_{\mathbb{Y}}(\bar{B})=\beta_{\mathbb{Y}}(\operatorname{con} v B)$, where $\bar{B}$ and $\operatorname{conv} B$ mean the closure and convex hull of $B$, respectively;

(3) $\beta_{\mathbb{Y}}(B) \leq \beta_{\mathbb{Y}}(C)$, where $B \subseteq C$;

(4) $\beta_{\mathbb{Y}}(B+C) \leq \beta_{\mathbb{Y}}(B)+\beta_{\mathbb{Y}}(C)$, where $B+C=\{x+y$ : $x \in B, y \in C\}$;

(5) $\beta_{\mathbb{Y}}(B \cup C) \leq \max \left\{\beta_{\mathbb{Y}}(B), \beta_{\mathbb{Y}}(C)\right\}$;

(6) $\beta_{\mathbb{Y}}(\lambda B) \leq|\lambda| \beta_{\mathbb{Y}}(B)$ for any $\lambda \in \mathbb{R}$;

(7) if the map $Q: D(Q) \subseteq \mathbb{Y} \rightarrow \mathbb{Z}$ is Lipschitz continuous with constant $k$, then $\beta_{\mathbb{Z}}(Q B) \leq k \beta_{\mathbb{Y}}(B)$ for any bounded subset $B \subseteq D(Q)$, where $\mathbb{Z}$ be a Banach space;

(8) $\beta_{\mathbb{Y}}(B)=\inf \left\{d_{\mathbb{Y}}(B, C) ; C \subseteq \mathbb{Y}\right.$ is precompact $\}=$ $\inf \left\{d_{\mathbb{Y}}(B, C) ; C \subseteq \mathbb{Y}\right.$ is finite valued $\}$, where $d_{\mathbb{Y}}(B, C)$ means the nonsymmetric (or symmetric) Hausdorff distance between $B$ and $C$ in $\mathbb{Y}$;

(9) if $\left\{W_{n}\right\}_{n=1}^{+\infty}$ is decreasing sequence of bounded closed nonempty subsets of $\mathbb{Y}$ and $\lim _{n \rightarrow \infty} \beta_{\mathbb{Y}}\left(W_{n}\right)=0$, then $\bigcap_{n=1}^{+\infty} W_{n}$ is nonempty and compact in $\mathbb{Y}$.

The map $Q: W \subseteq \mathbb{Y} \rightarrow \mathbb{Y}$ is said to be a $\beta_{\mathbb{Y} \text {-contraction }}$ if there exists a positive constant $k<1$ such that $\beta_{\mathbb{Y}}(Q(B)) \leq$ $k \beta_{\mathbb{Y}}(B)$ for any bounded closed subset $B \subseteq W$, where $\mathbb{Y}$ is a Banach space.

Lemma 3 (Darbo-Sadovskii [30]). If $W \subseteq Y$ is bounded closed and convex, the continuous map $Q: W \rightarrow W$ is a $\beta_{\mathbb{Y}}$-contraction, then the map $Q$ has at least one fixed point in $W$.

Lemma 4 (see [2]). If $W \subseteq P C([0, a] ; X)$ is bounded, then $\alpha(W(t)) \leq \alpha_{P C}(W)$ for all $t \in[0, a]$, where $W(t)=\{u(t)$ : $u \in W\} \subseteq X$. Furthermore if $W$ is equicontinuous on each interval $J_{i}$ of $[0, a]$, then $\alpha(W(t))$ is continuous on $[0, a]$, and $\alpha_{P C}(W)=\sup \{\alpha(W(t)): t \in[0, a]\}$.

Lemma 5 (see [3]). If $\left\{u_{n}\right\}_{n=1}^{\infty} \subset L^{1}(0, a ; X)$ is uniformly integrable, then $\alpha\left(\left\{u_{n}(t)\right\}_{n=1}^{\infty}\right)$ is measurable and

$$
\alpha\left(\left\{\int_{0}^{t} u_{n}(s) d s\right\}_{n=1}^{\infty}\right) \leq 2 \int_{0}^{t} \alpha\left(\left\{u_{n}(s)\right\}_{n=1}^{\infty}\right) d s .
$$

Lemma 6 (see [31]). If the semigroup $T(t)$ is equicontinuous and $\eta \in L^{1}\left(0, a ; R^{+}\right)$, then the set

$$
\begin{gathered}
\left\{t \longrightarrow \int_{0}^{t} T(t-s) u(s) d s ; u \in L^{1}\left(0, K ; R^{+}\right),\right. \\
\|u(s)\| \leq \eta(s), \text { for a.e } s \in[0, a]\}
\end{gathered}
$$

is equicontinuous on $[0, a]$. 
Lemma 7 (see [23]). If $W$ is bounded, then for each $\varepsilon>$ 0 , there is a sequence $\left\{u_{n}\right\}_{n=1}^{\infty} \subseteq W$, such that $\alpha(W) \leq$ $2 \alpha\left(\left\{u_{n}\right\}_{n=1}^{\infty}\right)+\varepsilon$.

\section{3. $g$ Is Compact}

In this section, we give the existence results of nonlocal integrodifferential equation (39). Here we list the following hypotheses.

$\left(H g_{1}\right)$ The $c_{0}$ semigroup $T(t), 0 \leq t \leq a$, generated by $A$ is equicontinuous.

$\left(H g_{2}\right) \quad(\mathrm{i}) \mathrm{g}: \mathrm{PC}([0, a] ; X) \rightarrow X$ is continuous and compact.

(ii) There exists $M>0$ such that $\|g(u)\| \leq$ $M$, for all $u \in \operatorname{PC}([0, a] ; X)$, and $\widehat{k}(s)=$ $\max \left\{1, \int_{0}^{s} k(s, \tau) d \tau\right\}$.

(I) Let $I_{i}: X \rightarrow X$ be continuous, compact map and there are nondecreasing functions $l_{i}: R^{+} \rightarrow R^{+}$, satisfying $\left\|I_{i}(x)\right\| \leq l_{i}(\|x\|), i=1,2, \ldots, p$.

$\left(H f_{1}\right)$ There exists a continuous function $a_{k}:[0, a] \times$ $[0, a] \rightarrow[0, \infty)$ and a nondecreasing continuous function $\Omega_{k}: R^{+} \rightarrow R^{+}$such that $\|k(t, s, x)\| \leq$ $a_{k}(t, s) \Omega_{k}(\|x\|)$ for all $x \in X$ a.e. $t, s \in[0, a]$. And there exists at least one mild solution to the following scalar equation:

$$
\begin{aligned}
m(t)= & M N \\
& +M \int_{0}^{t} a_{f}(s) \Omega_{f}\left(m(s), a_{k}(t, s) \Omega_{k} m(s),\right. \\
& \left.b_{h}(t, s) \Omega_{h} m(s)\right) d s \\
& +M \sum_{i=1}^{p} l_{i}(m(t)), \quad t \in[0, a] .
\end{aligned}
$$

$\left(H f_{2}\right) \quad$ (i) $f(\cdot, \cdot, x, y)$ is measurable for $x, y \in X, f(t, \cdot, \cdot, \cdot)$ is continuous for a.e. $t \in[0, a]$.

(ii) There exist a function $a_{f}(\cdot) \in L^{1}\left(0, a, R^{+}\right)$ and an increasing continuous function $\Omega_{f}$ : $R^{+} \rightarrow R^{+}$such that $\|f(t, x, y, z)\| \leq$ $a_{f}(t) \Omega_{f}(\|x\|,\|y\|,\|z\|)$ for all $x, y, z \in X$ and a.e. $t \in[0, a]$.

(iii) $f:[0, a] \times X \times X \rightarrow X$ is compact.

$\left(H f_{3}\right)$ There exists a function $\eta \in L^{1}\left(0, a ; R^{+}\right)$such that for any bounded $D \subset X$,

$$
\beta\left(f\left(t, D_{1}, D_{2}, D_{3}\right)\right) \leq \eta(t)\left(\beta\left(D_{1}\right)+\beta\left(D_{2}\right)+\beta\left(D_{3}\right)\right)
$$

for a.e. $t \in[0, a]$ and for any bounded subset $D \subset$ $\operatorname{PC}([0, a], X)$.

Here we let $\widehat{k}(s)=\int_{0}^{s} k(s, \tau) d \tau$ and $\left(1+2 \widehat{k_{1}}(s)+2 \widehat{k_{2}}(s)\right) \leq Q$.

Theorem 8. Assume that the hypotheses $\left(\mathrm{Hg}_{1}\right),\left(\mathrm{Hg}_{2}\right), \mathrm{I}$, $\left(H f_{1}\right)$, and $\left(H f_{2}\right)$ are satisfied; then the nonlocal impulsive problem (1) has at least one mild solution.
Proof. Let $m(t)$ be a solution of the scalar equation (5); the $\operatorname{map} K: \mathrm{PC}([0, a] ; X) \rightarrow \mathrm{PC}([0, a] ; X)$ is defined by

$$
(K u)(t)=\left(K_{1} u\right)(t)+\left(K_{2} u\right)(t)
$$

with

$$
\begin{aligned}
&\left(K_{1} u\right)(t)= T(t) g(u) \\
&+\int_{0}^{t} T(t-s) f\left(s, u(s), \int_{0}^{s} k(s, \tau, u(\tau)) d \tau,\right. \\
&\left.\int_{0}^{a} h(s, \tau, u(\tau)) d \tau\right) \\
&\left(K_{2} u\right)(t)=\sum_{0<t_{i}<t} T\left(t-t_{i}\right) I_{i}\left(u\left(t_{i}\right)\right),
\end{aligned}
$$

for all $t \in[0, a]$.

It is easy to see that the fixed point of $K$ is the mild solution of nonlocal impulsive problem (1).

From our hypotheses, the continuity of $K$ is proved as follows.

For this purpose, we assume that $u_{n} \rightarrow u$ in $\operatorname{PC}([0, a] ; X)$. It comes from the continuity of $k$ and $h$ that $k\left(s, \tau, u_{n}(\tau)\right) \rightarrow k(s, \tau, u(\tau))$ and $h\left(s, \tau, u_{n}(\tau)\right) \rightarrow$ $h(s, \tau, u(\tau))$, respectively.

By Lebesgue convergence theorem,

$$
\begin{aligned}
& \int_{0}^{s} k\left(s, \tau, u_{n}(\tau)\right) d \tau \longrightarrow \int_{0}^{s} k(s, \tau, u(\tau)) d \tau, \\
& \int_{0}^{a} h\left(s, \tau, u_{n}(\tau)\right) d \tau \longrightarrow \int_{0}^{a} h(s, \tau, u(\tau)) d \tau .
\end{aligned}
$$

Similarly we have

$$
\begin{aligned}
& f\left(s, u_{n}(s), \int_{0}^{s} k\left(s, \tau, u_{n}(\tau)\right) d \tau, \int_{0}^{a} h\left(s, \tau, u_{n}(\tau)\right) d \tau\right) \\
& \quad \longrightarrow f\left(s, u(s), \int_{0}^{s} k(s, \tau, u(\tau)) d \tau, \int_{0}^{a} h(s, \tau, u(\tau)) d \tau\right),
\end{aligned}
$$

for all $s \in[0, a]$. Consider

$$
\begin{aligned}
& \left\|K u_{n}-K u\right\| \\
& \leq N\left\|g\left(u_{n}\right)-g(u)\right\| \\
& \quad+N \int_{0}^{t}\left(\| f\left(s, u_{n}(s), \int_{0}^{s} k\left(s, \tau, u_{n}(\tau)\right) d \tau,\right.\right.
\end{aligned}
$$




$$
\begin{gathered}
\left.\int_{0}^{a} h\left(s, \tau, u_{n}(\tau)\right) d \tau\right) \\
-f\left(s, u(s), \int_{0}^{s} k(s, \tau, u(\tau)) d \tau,\right. \\
\left.\left.\int_{0}^{a} h(s, \tau,(\tau)) d \tau\right) \|\right) d s \\
+\sum_{i=1}^{p} M\left\|I_{i}\left(u_{n}\left(t_{i}\right)\right)-I_{i}\left(u\left(t_{i}\right)\right)\right\| \rightarrow 0,
\end{gathered}
$$

as $n \rightarrow \infty$. So $K u_{n} \rightarrow K u$ in $\mathrm{PC}([0, a] ; X)$. That is, $K$ is continuous.

We denote $W_{0}=\{u \in \mathrm{PC}([0, \mathrm{a}]) ; \mathrm{X}),\|u(t)\| \leq m(t)$ for all $t \in[0, a]\}$; then $W \subseteq \mathrm{PC}([o, a] ; X)$ is bounded and convex.

Define $W_{1}=\overline{\operatorname{conv}} K\left(W_{0}\right)$, where $\overline{\operatorname{conv}}$ means that the closure of the convex hull in $\operatorname{PC}([0, a] ; X)$.

For any $u \in K\left(W_{0}\right)$, we know that

$\|(u)(t)\| \leq M N$

$$
\begin{gathered}
+N \int_{0}^{t} a_{f}(s) \Omega_{f}\left(m(s), a_{k}(t, s) \Omega_{k} m(s),\right. \\
\left.b_{h}(t, s) \Omega_{h} m(s)\right) d s \\
+M \sum_{i=1}^{p} l_{i}(m(t)), \quad t \in[0, a],
\end{gathered}
$$

and by $\left(H f_{2}\right), W_{1} \subset W_{0}$.

From the Arzela-Ascoli theorem, to prove the compactness of $K$, we can prove that $K_{1} u: u \in W_{0}$ is equicontinuous and $K_{1} u(t) \subset X$ is precompact for $t \in[0, a]$ :

$$
\begin{aligned}
& \left\|K_{1} u(t+\sigma)-K_{1} u(t)\right\| \\
& =\| T(t+\sigma) g(u) \\
& \quad+\int_{0}^{t+\sigma} T(t+\sigma-s) f\left(s, u(s), \int_{0}^{s} k(s, \tau, u(\tau)) d \tau,\right. \\
& \quad-\left[T(t) g(u)+\int_{0}^{a} T(t-s) f\left(s, u(s), \int_{0}^{s} k(s, \tau, u(\tau)) d \tau, \tau(\tau)\right) d \tau\right) d s \\
& \left.\left.\quad\|[T(t+\sigma)-T(u)] g(u)\| \int_{0}^{a} h(s, \tau, u(\tau)) d \tau\right) d s\right] \| \\
& +\int_{t}^{t+\sigma}\|T(t+\sigma-s)\| \| f\left(s, u(s), \int_{0}^{s} k(s, \tau, u(\tau)) d \tau,\right. \\
& \left.\quad \int_{0}^{a} h(s, \tau, u(\tau)) d \tau\right) \| d s
\end{aligned}
$$

$$
\begin{aligned}
& +\int_{0}^{t}\|T(t+\sigma-s)-T(t-s)\| \\
& \times \| f\left(s, u(s), \int_{0}^{s} k(s, \tau, u(\tau)) d \tau,\right. \\
& \left.\qquad \int_{0}^{a} h(s, \tau, u(\tau)) d \tau\right) \| d s \\
& +N \int_{0}^{t+\sigma} a_{f}(s) \Omega_{f}\left(\|u(s)\|, \int_{0}^{s} a_{k}(s, \tau) \Omega_{k}(\|u(s)\|) d \tau,\right. \\
& +N \int_{0}^{t} \|[T(\sigma)-I] f\left(s, u(s), \int_{0}^{s} k(s, \tau, u(\tau)) d \tau,\right. \\
& \left.\quad b_{h}^{a}(s, \tau) \Omega_{h}(\|u(s)\|) d \tau\right) d s \\
& \left.\qquad \int_{0}^{a} h(s, \tau, u(\tau)) d \tau\right) \| d s .
\end{aligned}
$$

Since $f$ is compact, $M N\|T(\sigma)-I\|$ and $\|[T(\sigma)-$ $I] f\left(s, u(s), \int_{0}^{s} k(s, \tau, u(\tau)) d \tau, \int_{0}^{a} h(s, \tau, u(\tau)) d \tau\right) \| \rightarrow 0$ as $\sigma \rightarrow 0$ uniformly for $s \in[0, a]$ and $u \in \mathrm{PC}([0, a] ; X)$. This implies that for any $\varepsilon_{1}>0$ and $\varepsilon_{2}>0$, there exist a $\delta>0$ such that

$$
\begin{aligned}
& \|[T(\sigma)-I]\| \leq \varepsilon_{1}, \\
& \|[T(\sigma)-I] \\
& \quad \times f\left(s, u(s), \int_{0}^{s} k(s, \tau, u(\tau)) d \tau, \int_{0}^{a} h(s, \tau, u(\tau)) d \tau\right) \| \\
& \leq \varepsilon_{2},
\end{aligned}
$$

for $0 \leq \sigma<\delta$ and all $u \in \operatorname{PC}([0, a] ; X)$. Therefore

$$
\begin{aligned}
& \|[T(\sigma)-I]\| \\
& +\|[T(\sigma)-I] \\
& \quad \times f\left(s, u(s), \int_{0}^{s} k(s, \tau, u(\tau)) d \tau, \int_{0}^{a} h(s, \tau, u(\tau)) d \tau\right) \|
\end{aligned}
$$$$
\leq \varepsilon_{1}+\varepsilon_{2}=\varepsilon
$$

We know that

$$
\begin{array}{r}
\left\|K_{1} u(t+\sigma)-K_{1} u(t)\right\| \\
\leq M N \varepsilon+N \Omega_{f}\left(m(s),\left\|a_{k}(a, a)\right\|, \Omega_{k}(m(s)),\right. \\
\left.\left\|b_{h}(a, a)\right\|, \Omega_{h}(m(s))\right)+N \varepsilon,
\end{array}
$$

for $0 \leq \sigma<\delta$ and all $u \in \operatorname{PC}([0, a] ; X)$. So $\left\{K_{1} u: u \in W_{0}\right\}$ is equicontinuous. 
The set $\left\{T(t-s) f\left(s, u(s), \int_{0}^{s} k(s, \tau, u(\tau)) d \tau, \int_{0}^{a} h(s\right.\right.$, $\tau, u(\tau)) d \tau) ; t, s \in[0, a], u \in \operatorname{PC}([0, a] ; X)\}$ is precompact as $f$ is compact and $T(\cdot)$ is a $C_{0}$-semigroup.

So $K_{1} u(t) \subset X$ is precompact as $K_{1} u(t) \subset t \overline{\operatorname{conv}}\{T(t-$ s) $f\left(s, u(s), \int_{0}^{s} k(s, \tau, u(\tau)) d \tau, \int_{0}^{a} h(s, \tau, u(\tau)) d \tau\right) ; s \in[0, t]$, $u \in \operatorname{PC}([0, a] ; X)\}$ for all $t \in[0, a] . W_{1}$ is equicontinuous on each interval $J_{i}$ of $[0, a]$. For $t_{i} \leq t<t+\sigma \leq t_{i+1}, i=1,2, \ldots, p$, we have, using the semigroup properties

$$
\begin{aligned}
& \left\|\left(K_{2} u\right)(t+\sigma)-\left(K_{2} u\right)(t)\right\| \\
& \leq \| \sum_{0<t_{i}<t+\sigma} T\left(t+\sigma-t_{i}\right) I_{i}\left(x\left(t_{i}\right)\right) \\
& \quad-\sum_{0<t_{i}<t} T\left(t+\sigma-t_{i}\right) I_{i}\left(x\left(t_{i}\right)\right) \| \\
& +\| \sum_{0<t_{i}<t} T\left(t+\sigma-t_{i}\right) I_{i}\left(x\left(t_{i}\right)\right) \\
& \quad-\sum_{0<t_{i}<t} T\left(t-t_{i}\right) I_{i}\left(x\left(t_{i}\right)\right) \| \\
& \leq \sum_{0<t_{i}<t+\sigma}\left\|T\left(t+\sigma-t_{i}\right) I_{i}\left(x\left(t_{i}\right)\right)\right\| \\
& \quad+\sum_{0<t_{i}<t}\left\|T\left(t+\sigma-t_{i}\right) I_{i}\left(x\left(t_{i}\right)\right)-T\left(t-t_{i}\right) I_{i}\left(x\left(t_{i}\right)\right)\right\|,
\end{aligned}
$$

which follows that $\left\{K_{2} u: u \in W_{0}\right\}$ is equicontinuous on each $J_{i}$ due to the equicontinuous of $T(t)$ and hypotheses $(I)$. Therefore, $W_{1} \subset \mathrm{PC}([0, a] ; X)$ is bounded closed convex nonempty and equicontinuous on each interval $J_{i}, i=$ $0,1,2, \ldots, p$.

We define $W_{n+1}=\overline{\operatorname{conv}} K\left(W_{n}\right)$, for $n=1,2, \ldots, p$. From above we know that $\left\{W_{n}\right\}_{n=1}^{\infty}$ is a decreasing sequence of bounded, closed, convex nonempty subsets in $\mathrm{PC}([0, a] ; X)$ and equicontinuous on each $J_{i}, i=1,2, \ldots, p$.

Now for $n \geq 1$ and $t \in[0, a], W_{n}(t)$ and $K\left(W_{n}(t)\right)$ are bounded subsets of $X$. Hence for any $\varepsilon>0$, there is a sequence $\left\{u_{k}\right\}_{k=1}^{\infty} \subset W_{n}$ such that (see, e.g., [2, page 125])

$$
\begin{aligned}
& \beta\left(W_{n+1}(t)\right) \\
& =\beta\left(K W_{n}(t)\right) \\
& \leq 2 \beta\left(T(t) g\left(\left\{u_{k}\right\}_{k=1}^{\infty}\right)\right) \\
& \quad+2 \beta\left(\int _ { 0 } ^ { t } T ( t - s ) f \left(s,\left\{u_{k}(s)\right\}_{k=1}^{\infty}, \int_{0}^{s} k\left(s, \tau,\left\{u_{k}(\tau)\right\}_{k=1}^{\infty}\right) d \tau,\right.\right. \\
& \left.\left.\qquad \int_{0}^{a} h\left(s, \tau,\left\{u_{k}(\tau)\right\}_{k=1}^{\infty}\right) d \tau\right) d s\right) \\
& \quad+2 \beta\left(\sum_{i=1}^{p} T\left(t-t_{i}\right) I_{i}\left\{u_{k}\left(t_{i}\right)\right\}_{k=1}^{\infty}\right)+\varepsilon,
\end{aligned}
$$

for $t \in[0, a]$.
From the compactness of $g$ and $I_{i}$, by Lemmas 2 and 5 and $\left(H f_{3}\right)$, we have

$$
\begin{aligned}
& \beta\left(W_{n+1}(t)\right) \\
& \leq 2 \beta\left(\int _ { 0 } ^ { t } T ( t - s ) f \left(s,\left\{u_{k}(s)\right\}_{k=1}^{\infty}, \int_{0}^{s} k\left(s, \tau,\left\{u_{k}(\tau)\right\}_{k=1}^{\infty}\right) d \tau,\right.\right. \\
& \left.\left.\int_{0}^{a} h\left(s, \tau,\left\{u_{k}(\tau)\right\}_{k=1}^{\infty}\right) d \tau\right) d s\right)+\varepsilon \\
& \leq 4 N \int_{0}^{t} \beta\left(f \left(s,\left\{u_{k}(s)\right\}_{k=1}^{\infty}, \int_{0}^{s} k\left(s, \tau,\left\{u_{k}(\tau)\right\}_{k=1}^{\infty}\right) d \tau,\right.\right. \\
& \left.\left.\int_{0}^{a} h\left(s, \tau,\left\{u_{k}(\tau)\right\}_{k=1}^{\infty}\right) d \tau\right)\right) d s+\varepsilon \\
& \leq 4 N \int_{0}^{t} \eta(s)\left(\beta\left(\left\{u_{k}(s)\right\}_{k=1}^{\infty}\right)+\beta\left(\int_{0}^{s} k\left(s, \tau,\left\{u_{k}(\tau)\right\}_{k=1}^{\infty}\right) d \tau\right)\right. \\
& \left.+\beta\left(\int_{0}^{a} h\left(s, \tau,\left\{u_{k}(\tau)\right\}_{k=1}^{\infty}\right) d \tau\right)\right) d s+\varepsilon \\
& \leq 4 N \int_{0}^{t} \eta(s)\left(\beta\left(\left\{u_{k}(s)\right\}_{k=1}^{\infty}\right)\right. \\
& +2 \int_{0}^{s} \beta\left(k\left(s, \tau,\left\{u_{k}(\tau)\right\}_{k=1}^{\infty}\right) d \tau\right) \\
& \left.+2 \int_{0}^{a} \beta\left(h\left(s, \tau,\left\{u_{k}(\tau)\right\}_{k=1}^{\infty}\right) d \tau\right)\right) d s+\varepsilon \\
& \leq 4 N \int_{0}^{t} \eta(s)\left(\beta\left(\left\{u_{k}(s)\right\}_{k=1}^{\infty}\right)\right. \\
& +2\left(\int_{0}^{s} \eta_{1}(s, \tau) d \tau\right) \beta\left(\left\{u_{k}(s)\right\}_{k=1}^{\infty}\right) \\
& \left.+2\left(\int_{0}^{a} \eta_{2}(s, \tau) d \tau\right) \beta\left(\left\{u_{k}(s)\right\}_{k=1}^{\infty}\right)\right) d s+\varepsilon \\
& \leq 4 N \int_{0}^{t} \eta(s)\left(\beta\left(\left\{u_{k}(s)\right\}_{k=1}^{\infty}\right)+2 \widehat{k_{1}}(s) \beta\left(\left\{u_{k}(s)\right\}_{k=1}^{\infty}\right)\right. \\
& \left.+2 \widehat{k_{2}}(s) \beta\left(\left\{u_{k}(s)\right\}_{k=1}^{\infty}\right)\right) d s+\varepsilon \\
& \leq 4 N \int_{0}^{t} \eta(s)\left(\beta\left(W_{n}(s)\right)+2 \widehat{k_{1}}(s) \beta\left(W_{n}(s)\right)\right. \\
& \left.+2 \widehat{k_{2}}(s) \beta\left(W_{n}(s)\right)\right) d s+\varepsilon \\
& \leq 4 N \int_{0}^{t} \eta(s) \beta\left(W_{n}(s)\right)\left(1+2 \widehat{k_{1}}(s)+2 \widehat{k_{2}}(s)\right) d s+\varepsilon \\
& \leq 4 N Q \int_{0}^{t} \eta(s) \beta\left(W_{n}(s)\right) d s+\varepsilon .
\end{aligned}
$$

Since $\varepsilon>0$ is arbitrary, it follows from the above inequality that

$$
\beta\left(W_{n+1}(t)\right) \leq 4 N Q \int_{0}^{t} \eta(s) \beta_{\mathrm{PC}}\left(W_{n}(s)\right) d s,
$$


for all $t \in[0, a]$. Since $W_{n}$ is decreasing for $n$, we define $f_{n}(t)=$ $\lim _{n \rightarrow \infty} \beta\left(W_{n}(t)\right)$ for all $t \in[0, a]$. From $(20)$, we have

$$
f_{n+1}(t) \leq 4 N Q \int_{0}^{t} \eta(s) f_{n}(s) d s,
$$

for $t \in[0, a]$, which implies that $f_{n}(t)=0$ for all $t \in[0, a]$. By Lemma 4, we know that

$$
\lim _{n \rightarrow \infty} \beta_{\text {PC }}\left(W_{n}\right)=0 .
$$

Using Lemma 2, we also know that

$$
W=\bigcap_{n=1}^{\infty} W_{n}
$$

is convex, compact, and nonempty in $\operatorname{PC}([0, a] ; X)$ and $K(W) \subset W$.

By the famous Schauder's fixed point theorem, there exists at least one mild solution $u$ of the problem (1), where $u \in W$ is a fixed point of the continuous map $K$.

\section{4. $g$ Is Lipschitz}

In this section, we discuss the problem (1) when $g$ is Lipschitz continuous and $I_{i}, i=1,2, \ldots, p$ is not compact. We replace hypotheses $\left(H f_{2}\right),(I)$ by

$\left(H f_{2}\right)^{\prime}$ There is a constant $L \in(0,1 / M)$ such that $\| g(u)-$ $g(v)\|\leq L\| u-v \|_{\mathrm{PC}}$ for all $u, v \in \mathrm{PC}([0, a] ; X)$.

(I') There exists $L_{i}>0, i=1,2, \ldots, p$, such that $\| I_{i}(u)-$ $I_{i}(v)\left\|\leq L_{i}\right\| u-v \|$, for all $u, v \in X$.

Theorem 9. Assume that the hypotheses $\left(H g_{1}\right),\left(H f_{2}\right)^{\prime},\left(I^{\prime}\right)$, $\left(H f_{1}\right)-\left(H f_{3}\right)$ are satisfied. Then the nonlocal impulsive problem (1) has at least one mild solution on [0,a], provided that

$$
M L+4 N Q \int_{0}^{t} \eta(s) d s+2 N \sum_{i=1}^{p} L_{i}<1 .
$$

Proof. Define the operator $K \quad: \quad \operatorname{PC}([0, a] ; X) \quad \rightarrow$ $\operatorname{PC}([0, a] ; X)$ by

$$
(K u)(t)=\left(K_{1} u\right)(t)+\left(K_{2} u\right)(t)+\left(K_{3} u\right)(t) .
$$

With

$$
\begin{aligned}
& \left(K_{1} u\right)(t)=T(t) g(u), \\
& \left(K_{2} u\right)(t)=\int_{0}^{t} T(t-s) f\left(s, u(s), \int_{0}^{s} k(s, \tau, u(\tau)) d \tau,\right. \\
& \left.\qquad \int_{0}^{a} h(s, \tau, u(\tau)) d \tau\right) d s, \\
& \left(K_{3} u\right)(t)=\sum_{0<t_{i}<t} T\left(t-t_{i}\right) I_{i}\left(u\left(t_{i}\right)\right),
\end{aligned}
$$

for all $u \in \mathrm{PC}([0, a] ; X)$.

Define $W_{0}=\{u \in \operatorname{PC}([0, a] ; X)\}:\|u(t)\| \leq m(t)$ for all $t \in[0, a]$, and let $W=\overline{K W_{0}}$.
Then from the proof of Theorem 8 , we know that $W$ is a bounded closed convex and equicontinuous subset of $\mathrm{PC}([0, a] ; X)$ and $K W \subset W$. We will prove that $K$ is $\beta_{\mathrm{PC}}$-contraction on $W$. Then Darbo-Sadovskii fixed point theorem can be used to get a fixed point of $K$ in $W$, which is a mild solution of (1).

We first show that $K_{1}$ is Lipschitz on $\operatorname{PC}([0, a] ; X)$.

In fact, take $u, v \in \mathrm{PC}([0, a] ; X)$ arbitrary. Then by $\left(H f_{2}\right)^{\prime}$, we have

$$
\begin{aligned}
& \left\|\left(K_{1} u\right)(t)-\left(K_{2} u\right)(t)\right\| \\
& \quad \leq M\|g(u)-g(v)\| \leq M L\|u-v\|_{\mathrm{PC}} \quad \forall t \in[0, a] .
\end{aligned}
$$

It follows that $\left\|K_{1} u-K_{1} v\right\| \leq M L\|u-v\|_{\mathrm{PC}}$ for all $u, v \in$ $\operatorname{PC}([0, a] ; X)$. That is, $K_{1}$ is Lipschitz with Lipschitz constant $M L$.

Next, for every bounded subset $B \subset W$, for any $\varepsilon>0$, there is a sequence $\left\{u_{k}\right\}_{k=1}^{\infty} \subset B$ such that $\beta\left(K_{2} B(t)\right) \leq$ $2 \beta\left(\left\{K_{2} u_{k}(t)\right\}_{k=1}^{\infty}\right)+\varepsilon$, for $t \in[0, a]$. Since $B$ and $K_{2} B$ are equicontinuous, we get from Lemmas 2 and 5 and $\left(\mathrm{Hf}_{3}\right)$ that

$$
\begin{aligned}
& \beta\left(K_{2} B(t)\right) \\
& \leq 2 \beta\left(\int _ { 0 } ^ { t } T ( t - s ) f \left(s,\left\{u_{k}(s)\right\}_{k=1}^{\infty}, \int_{0}^{s} k\left(s, \tau,\left\{u_{k}(\tau)\right\}_{k=1}^{\infty}\right) d \tau,\right.\right. \\
& \left.\left.\int_{0}^{a} h\left(s, \tau,\left\{u_{k}(\tau)\right\}_{k=1}^{\infty}\right) d \tau\right) d s\right)+\varepsilon \\
& \leq 4 N \int_{0}^{t} \beta\left(f \left(s,\left\{u_{k}(s)\right\}_{k=1}^{\infty}, \int_{0}^{s} k\left(s, \tau,\left\{u_{k}(\tau)\right\}_{k=1}^{\infty}\right) d \tau,\right.\right. \\
& \left.\left.\int_{0}^{a} h\left(s, \tau,\left\{u_{k}(\tau)\right\}_{k=1}^{\infty}\right) d \tau\right)\right) d s+\varepsilon \\
& \leq 4 N \int_{0}^{t} \eta(s)\left(\beta\left(\left\{u_{k}(s)\right\}_{k=1}^{\infty}\right)+\beta\left(\int_{0}^{s} k\left(s, \tau,\left\{u_{k}(\tau)\right\}_{k=1}^{\infty}\right) d \tau\right)\right. \\
& \left.+\beta\left(\int_{0}^{a} h\left(s, \tau,\left\{u_{k}(\tau)\right\}_{k=1}^{\infty}\right) d \tau\right)\right) d s+\varepsilon \\
& \leq 4 N \int_{0}^{t} \eta(s)\left(\beta\left(\left\{u_{k}(s)\right\}_{k=1}^{\infty}\right)+2 \int_{0}^{s} \beta\left(k\left(s, \tau,\left\{u_{k}(\tau)\right\}_{k=1}^{\infty}\right) d \tau\right)\right. \\
& \left.+2 \int_{0}^{a} \beta\left(h\left(s, \tau,\left\{u_{k}(\tau)\right\}_{k=1}^{\infty}\right) d \tau\right)\right) d s+\varepsilon \\
& \leq 4 N \int_{0}^{t} \eta(s)\left(\beta\left(\left\{u_{k}(s)\right\}_{k=1}^{\infty}\right)\right. \\
& +2\left(\int_{0}^{s} \eta_{1}(s, \tau) d \tau\right) \beta\left(\left\{u_{k}(s)\right\}_{k=1}^{\infty}\right)
\end{aligned}
$$




$$
\begin{array}{r}
\left.+2\left(\int_{0}^{a} \eta_{2}(s, \tau) d \tau\right) \beta\left(\left\{u_{k}(s)\right\}_{k=1}^{\infty}\right)\right) d s+\varepsilon \\
\leq 4 N \int_{0}^{t} \eta(s)\left(\beta\left(\left\{u_{k}(s)\right\}_{k=1}^{\infty}\right)+2 \widehat{k_{1}}(s) \beta\left(\left\{u_{k}(s)\right\}_{k=1}^{\infty}\right)\right. \\
\left.+2 \widehat{k_{2}}(s) \beta\left(\left\{u_{k}(s)\right\}_{k=1}^{\infty}\right)\right) d s+\varepsilon \\
\leq 4 N \int_{0}^{a} \eta(s)\left(\beta_{\mathrm{PC}}\left(\left\{u_{k}\right\}_{k=1}^{\infty}\right)+2 \widehat{k_{1}}(s) \beta_{\mathrm{PC}}\left(\left\{u_{k}\right\}_{k=1}^{\infty}\right)\right. \\
\left.+2 \widehat{k_{2}}(s) \beta_{\mathrm{PC}}\left(\left\{u_{k}\right\}_{k=1}^{\infty}\right)\right) d s+\varepsilon \\
\leq 4 N \int_{0}^{a} \eta(s) \beta_{\mathrm{PC}}(B)\left(1+2 \widehat{k_{1}}(s)+2 \widehat{k_{2}}(s)\right) d s+\varepsilon \\
\leq 4 N Q \int_{0}^{a} \eta(s) d s \beta_{\mathrm{PC}}(B)+\varepsilon,
\end{array}
$$

for $t \in[0, a]$. Since $\varepsilon>0$ arbitrary, we have

$$
\beta_{\mathrm{PC}}\left(K_{2} B\right) \leq 4 N Q \int_{0}^{a} \eta(s) \beta_{\mathrm{PC}}(B) d s,
$$

for any bounded subset $B \subset W$.

$$
\begin{aligned}
\beta\left(K_{3} B(t)\right) & \leq 2 N \sum_{i=1}^{p} \beta\left(I_{i}\left\{u_{k}\left(t_{i}\right)\right\}_{k=1}^{\infty}\right) \\
& \leq 2 N \sum_{i=1}^{p} L_{i} \beta\left(\left\{u_{k}\left(t_{i}\right)\right\}_{k=1}^{\infty}\right) \\
\beta_{\mathrm{PC}}\left(K_{3} B\right) & \leq 2 N \sum_{i=1}^{p} L_{i} \beta_{\mathrm{PC}}(B),
\end{aligned}
$$

for any subset $B \subset W$; due to Lemma 2, (29), and (30), we have

$$
\begin{aligned}
\beta_{\mathrm{PC}} & (K B) \\
& =\beta_{\mathrm{PC}}\left(K_{1} B\right)+\beta_{\mathrm{PC}}\left(K_{2} B\right)+\beta_{\mathrm{PC}}\left(K_{3} B\right) \\
& \leq M L \beta_{\mathrm{PC}}(B)+4 N Q \int_{0}^{a} \eta(s) \beta_{\mathrm{PC}}(B) d s+2 N \sum_{i=1}^{p} L_{i} \beta_{\mathrm{PC}}(B) \\
& \leq\left(M L+4 N Q \int_{0}^{a} \eta(s) d s+2 N \sum_{i=1}^{p} L_{i}\right) \beta_{\mathrm{PC}}(B) .
\end{aligned}
$$

From (24), we know that $K$ is $\beta_{\mathrm{PC}}$-contraction on $W$. By Lemma 3 , there is a fixed point $u$ of $K$ in $W$, which is a mild solution of problem (1).

\section{Classical Solutions}

To study the classical solutions, let us recall the following result.
Lemma 10. Assume that $u_{0} \in D(A), q_{i} \in D(A), i=$ $1,2, \ldots, p$, and that $f \in C^{1}([0, a] \times X, X)$.

Then the impulsive differential equation

$$
\begin{aligned}
u^{\prime}(t) & =A u(t)+f(t, u(t)), \quad t \in[0, a], t \neq t_{i} \\
u(0) & =u_{0}, \\
\Delta u(t) & =q_{i}, \quad i=1,2, \ldots, p,
\end{aligned}
$$

has a unique classical solution $u(\cdot)$ which satisfies, for $t \in[0, a]$,

$u(t)$

$$
=T(t) u(0)=\int_{0}^{t} T(t-s) f(s, u(s)) d s+\sum_{0<t_{i}<t} T\left(t-t_{i}\right) q_{i} .
$$

Now we make the following assumption.

$\left(H^{1}\right)$ There exists a function $\eta \in L^{1}\left(0, a ; R^{+}\right)$such that for any bounded $D \subset X$,

$$
\beta\left(f\left(t, D_{1}, D_{2}, D_{3}\right)\right) \leq \eta(t)\left(\beta\left(D_{1}\right)+\beta\left(D_{2}\right)+\beta\left(D_{3}\right)\right),
$$

or a.e. $t \in[0, a]$ and for any bounded subset $D \subset P C([0, a], X)$.

Theorem 11. Let $\left(H^{1}\right)$ be satisfied and $u(\cdot)$ a mild solution of the problem (1). Assume that $u(0) \in D(A), I_{i}\left(u\left(t_{i}\right)\right) \in D(A)$, $i=1,2, \ldots, p$ and that $f \in C^{1}([0, a] \times X, X)$. Then $u(0)$ gives rise to a classical solution of the problem (1).

If $u(\cdot)$ is a uniquely determined mild solution, then it gives rise to a unique classical solution.

Proof. We can define $q_{i}=I_{i}\left(u\left(t_{i}\right)\right), i=1,2, \ldots, p$.

From Lemma 10,

$$
\begin{gathered}
v^{\prime}(t)=A v(t)+f\left(t, v(t), \int_{0}^{t} k(t, s, v(s)) d s,\right. \\
\left.\int_{0}^{a} h(t, s, v(s)) d s\right) \quad t \in[0, a], t \neq t_{i} \\
v(0)=u_{0}+g(u), \\
\Delta v(t)=q_{i}, \quad i=1,2, \ldots, p
\end{gathered}
$$

has a unique classical solution $v(\cdot)$ which satisfies, for $t \in$ $[0, a]$,

$$
\begin{aligned}
v(t)= & T(t)\left[u_{0}-g(u)\right] \\
& +\int_{0}^{t} T(t-s) f\left(s, v(s), \int_{0}^{s} k(s, \tau, v(\tau)) d \tau,\right. \\
& \left.\quad \int_{0}^{a} h(s, \tau, v(\tau)) d \tau\right) d s \\
& +\sum_{0<t_{i}<t} T\left(t-t_{i}\right) I_{i}\left(u\left(t_{i}\right)\right), \quad 0 \leq t \leq a .
\end{aligned}
$$


Now, $u(\cdot)$ is a mild solution of the problem (1), so that we get or for $t \in[0, a]$,

$$
\begin{aligned}
u(t)= & T(t)\left[u_{0}-g(u)\right] \\
& +\int_{0}^{t} T(t-s) f\left(s, u(s), \int_{0}^{s} k(s, \tau, u(\tau)) d \tau,\right. \\
& \left.\int_{0}^{a} h(s, \tau, u(\tau)) d \tau\right) d s \\
& +\sum_{0<t_{i}<t} T\left(t-t_{i}\right) I_{i}\left(u\left(t_{i}\right)\right), \quad 0 \leq t \leq a .
\end{aligned}
$$

Thus we get

$$
\begin{aligned}
& v(t)-u(t) \\
&=\int_{0}^{t} T(t-s)\left(f \left(s, v(s), \int_{0}^{s} k(s, \tau, v(\tau)) d \tau\right.\right.\left.\int_{0}^{a} h(s, \tau, v(\tau)) d \tau\right) \\
&-f\left(s, u(s), \int_{0}^{s} k(s, \tau, u(\tau)) d \tau\right. \\
&\left.\left.\int_{0}^{a} h(s, \tau, u(\tau)) d \tau\right)\right) d s
\end{aligned}
$$

which gives, by $\left(H^{1}\right)$ and an application of Gronwall's inequality, $\|u-v\|_{\mathrm{PC}}=0$.

This implies that $u(\cdot)$ gives rise to a classical solution and completes the proof.

\section{Example}

Let $\Omega$ be a bounded domain in $R_{n}$ with smooth boundary $\partial \Omega$, and $X=L^{2}(\Omega)$. Consider the following nonlinear integrodifferential equation in $X$ :

$$
\begin{aligned}
\frac{\partial u(t, y)}{\partial t}= & \Delta u(t, y) \\
& +\frac{\gamma_{1} u(t, y)}{(1+t)\left(1+t^{2}\right)} \sin (u(t, y)) \\
& +\int_{0}^{t} \frac{\gamma_{2} u(s, y)}{(1+t)\left(1+t^{2}\right)(1+s)^{2}} d s \\
& +\int_{0}^{a} \frac{\gamma_{3} u(s, y)}{(1+t)\left(1+t^{2}\right)(1+s)^{2}\left(1+s^{2}\right)^{2}} d s, \\
u\left(t_{i}^{+}, y\right)- & u\left(t_{i}^{-}, y\right)=I_{i}\left(u\left(t_{i}, y\right)\right), \quad i=1,2, \ldots, p,
\end{aligned}
$$

with nonlocal conditions

$$
u(0)=u_{0}(y)+\int_{\Omega} \int_{0}^{a} h_{1}(t, y) \log \left(1+|u(t, s)|^{1 / 2}\right) d t d s,
$$$$
y \in \Omega \text {, }
$$

or

$$
u(0)=u_{0}(y)+\gamma_{4} u(t, y), \quad y \in \Omega
$$

where $\gamma_{1}, \gamma_{2}, \gamma_{3}, \gamma_{4}, \in R, h_{1}(t, y) \in \operatorname{PC}([0, a] ; \bar{\Omega})$. Set $A=$ $\Delta, D(A)=W^{2,2}(\Omega) \bigcap W_{0}^{1,2}(\Omega)$,

$$
\begin{aligned}
f(t, & \left.u(t), \int_{0}^{t} k(t, s, u(s)) d s, \int_{0}^{a} h(t, s, u(s)) d s\right)(y) \\
= & \frac{\gamma_{1} u(t, y)}{(1+t)\left(1+t^{2}\right)} \sin (u(t, y)) \\
& +\int_{0}^{t} \frac{\gamma_{2} u(s, y)}{(1+t)\left(1+t^{2}\right)(1+s)^{2}} d s \\
& +\int_{0}^{a} \frac{\gamma_{3} u(s, y)}{(1+t)\left(1+t^{2}\right)(1+s)^{2}\left(1+s^{2}\right)^{2}} d s .
\end{aligned}
$$

Define nonlocal conditions

$$
\begin{aligned}
& g(u)(y) \\
& \quad=u_{0}(y)+\int_{\Omega} \int_{0}^{a} h_{1}(t, y) \log \left(1+|u(t, s)|^{1 / 2}\right) d t d s
\end{aligned}
$$

or

$$
g(u)(y)=u_{0}(y)+\gamma_{4} u(t, y)
$$

It is easy to see that $A$ generates a compact $C_{0}$-semigroup in $X$, and

$$
\begin{array}{r}
\left\|f\left(t, u(t), \int_{0}^{t} k(t, s, u(s)) d s, \int_{0}^{a} h(t, s, u(s)) d s\right)\right\| \\
\leq|\gamma|(1,\|u\|,\|j\|,\|q\|), \\
\qquad|\gamma|=\max \left\{\left|\gamma_{1}\right|,\left|\gamma_{2}\right|,\left|\gamma_{3}\right|\right\},
\end{array}
$$

where

$$
\begin{gathered}
j=\int_{0}^{t} \frac{\gamma_{2} u(s, y)}{(1+t)\left(1+t^{2}\right)(1+s)^{2}} d s, \\
k(t, s, u(s))=\frac{\gamma_{2} u(s, y)}{(1+t)\left(1+t^{2}\right)(1+s)^{2}}, \\
q=\int_{0}^{a} \frac{\gamma_{3} u(s, y)}{(1+t)\left(1+t^{2}\right)(1+s)^{2}\left(1+s^{2}\right)^{2}} d s, \\
h(t, s, u(s))=\frac{\gamma_{3} u(s, y)}{(1+t)\left(1+t^{2}\right)(1+s)^{2}\left(1+s^{2}\right)^{2}},
\end{gathered}
$$

and $\|k(t, s, u(s))\| \leq\left|\gamma_{2}\right|(1+\|u\|),\|h(t, s, u(s))\| \leq\left|\gamma_{3}\right|(1+\|u\|)$, $\left\|I_{i}(u)\right\| \leq l_{i}(\|u\|), i=1,2, \ldots, p$.

For nonlocal conditions (44), $\|g(u)\| \leq a(\operatorname{mes}(\Omega))$ $\max _{t \in[0, a], y \in \Omega}\left|h_{1}(t, y)\right|\left[\|u\|+(\operatorname{mes}(\Omega))^{1 / 2}\right], u \in \operatorname{PC}([0, a] ; X)$, and $g$ is compact example of [18]. 
For nonlocal conditions (45),

$$
\left\|g\left(u_{1}\right)-g\left(u_{2}\right)\right\| \leq\left|\gamma_{4}\right|\left\|u_{1}-u_{2}\right\| .
$$

Hence, $g$ is Lipschitz. Furthermore, $\gamma_{1}, \gamma_{2}, \gamma_{3}, \gamma_{4}$ and $a$ can be chosen such that (24) is also satisfied. Obviously, it satisfies all the assumptions given in our Theorem 9; the problem has at least one mild solution in $\operatorname{PC}\left([0, a] ; L^{2}(\Omega)\right)$.

\section{Conflict of Interests}

The authors declare that there is no conflict of interests regarding the publication of this paper.

\section{Acknowledgment}

The authors are thankful for Project MTM2010-16499 from MEC of Spain.

\section{References}

[1] Q. Dong and G. Li, "Existence of solutions for semilinear differential equations with nonlocal conditions in Banach spaces," Electronic Journal of Qualitative Theory of Differential Equations, vol. 47, p. 13, 2009.

[2] T. Kato, "Quasi-linear equations of evolution, with applications to partial differential equations," in Spectral Theory and Differential Equations, vol. 448 of Lectures Notes in Mathematics, pp. 25-70, Springer, Berlin, Germany, 1975.

[3] T. Kato, "Abstract evolution equations, linear and quasilinear, revisited," in Functional Analysis and Related Topics, 1991 (Kyoto), vol. 1540 of Lectures Notes in Mathematics, pp. 103-125, Springer, Berlin, Germany, 1993.

[4] S. K. Ntouyas and P. Ch. Tsamatos, "Global existence for second order semilinear ordinary and delay integrodifferential equations with nonlocal conditions," Applicable Analysis, vol. 67, no. 3-4, pp. 245-257, 1997.

[5] Y. Yang and J. Wang, "On some existence results of mild solutions for nonlocal integrodifferential Cauchy problems in Banach spaces," Opuscula Mathematica, vol. 31, no. 3, pp. 443455, 2011.

[6] A. Pazy, Semigroups of Linear Operators and Applications to Partial Differential Equations, Springer, New York, NY, USA, 1983.

[7] X. Xue, "Semilinear nonlocal differential equations with measure of noncompactness in Banach spaces," Nanjing University. Journal. Mathematical Biquarterly, vol. 24, no. 2, pp. 264-276, 2007.

[8] C. Lizama and J. C. Pozo, "Existence of mild solutions for a semilinear integrodifferential equation with nonlocal initial conditions," Abstract and Applied Analysis, vol. 2012, Article ID 647103, 15 pages, 2012.

[9] N. U. Ahmed, "Systems governed by impulsive differential inclusions on Hilbert spaces," Nonlinear Analysis. Theory, Methods \& Applications A, vol. 45, no. 6, pp. 693-706, 2001.

[10] M. Benchohra, J. Henderson, and S. Ntouyas, Impulsive Differential Equations and Inclusions, vol. 2 of Contemporary Mathematics and Its Applications, Hindawi Publishing Corporation, New York, NY, USA, 2006.
[11] K. Malar and A. Tamilarasi, "Existence of mild solutions for nonlocal impulsive integrodifferential equations with the measure of noncompactness," Far East Journal of Applied Mathematics, vol. 57, no. 1, pp. 15-31, 2011.

[12] S. Migórski and A. Ochal, "Nonlinear impulsive evolution inclusions of second order," Dynamic Systems and Applications, vol. 16, no. 1, pp. 155-173, 2007.

[13] S. Ji and S. Wen, "Nonlocal Cauchy problem for impulsive differential equations in Banach spaces," International Journal of Nonlinear Science, vol. 10, no. 1, pp. 88-95, 2010.

[14] L. Byszewski and H. Akca, "Existence of solutions of a semilinear functional-differential evolution nonlocal problem," Nonlinear Analysis. Theory, Methods \& Applications A, vol. 34, no. 1, pp. 65-72, 1998.

[15] L. Byszewski, "Theorems about the existence and uniqueness of solutions of a semilinear evolution nonlocal Cauchy problem," Journal of Mathematical Analysis and Applications, vol. 162, no. 2, pp. 494-505, 1991.

[16] S. Aizicovici and M. McKibben, "Existence results for a class of abstract nonlocal Cauchy problems," Nonlinear Analysis. Theory, Methods \& Applications A, vol. 39, no. 5, pp. 649-668, 2000.

[17] J. Liang, J. Liu, and T.-J. Xiao, "Nonlocal Cauchy problems governed by compact operator families," Nonlinear Analysis. Theory, Methods \& Applications A, vol. 57, no. 2, pp. 183-189, 2004.

[18] J. Liang, J. H. Liu, and T.-J. Xiao, "Nonlocal problems for integrodifferential equations," Dynamics of Continuous, Discrete \& Impulsive Systems A, vol. 15, no. 6, pp. 815-824, 2008.

[19] L. Byszewski and V. Lakshmikantham, “Theorem about the existence and uniqueness of a solution of a nonlocal abstract Cauchy problem in a Banach space," Applicable Analysis, vol. 40, no. 1, pp. 11-19, 1991.

[20] S. K. Ntouyas and P. Ch. Tsamatos, "Global existence for semilinear evolution equations with nonlocal conditions," Journal of Mathematical Analysis and Applications, vol. 210, no. 2, pp. 679687, 1997.

[21] S. K. Ntouyas and P. Ch. Tsamatos, "Global existence for semilinear evolution integrodifferential equations with delay and nonlocal conditions," Applicable Analysis, vol. 64, no. 1-2, pp. 99-105, 1997.

[22] T. Zhu, C. Song, and G. Li, "Existence of mild solutions for abstract semilinear evolution equations in Banach spaces," Nonlinear Analysis. Theory, Methods \& Applications A, vol. 75, no. 1, pp. 177-181, 2012.

[23] J. H. Liu, "Nonlinear impulsive evolution equations," Dynamics of Continuous, Discrete and Impulsive Systems, vol. 6, no. 1, pp. 77-85, 1999.

[24] S. Ji and G. Li, "A unified approach to nonlocal impulsive differential equations with the measure of noncompactness," Advances in Difference Equations, vol. 2012, article 182, 2012.

[25] B. Ahmad, K. Malar, and K. Karthikeyan, "A study of nonlocal problems of impulsive integrodifferential equations with measure of noncompactness," Advances in Difference Equations, vol. 2013, article 205, 2013.

[26] S. Ji and G. Li, "Existence results for impulsive differential inclusions with nonlocal conditions," Computers \& Mathematics with Applications, vol. 62, no. 4, pp. 1908-1915, 2011.

[27] L. Zhu, Q. Dong, and G. Li, "Impulsive differential equations with nonlocal conditionsin general Banach spaces," Advances in Difference Equations, vol. 2012, article 10, 2012. 
[28] A. Debbouche, D. Baleanu, and R. P. Agarwal, "Nonlocal nonlinear integrodifferential equations of fractional orders," Boundary Value Problems, vol. 2012, article 78, 2012.

[29] D. Baleanu, K. Diethelm, E. Scalas, and J. J. Trujillo, Fractional Calculus Models and Numerical Methods, vol. 3 of Series on Complexity, Nonlinearity and Chaos, World Scientific, Singapore, 2012.

[30] J. Banaś and K. Goebel, Measures of Noncompactness in Banach Apaces, vol. 60 of Lecture Notes in Pure and Applied Mathematics, Marcel Dekker, New York, NY, USA, 1980.

[31] D. Both, "Multivalued perturbation of $\mathrm{m}$-accretive differential inclusions," Israel Journal of Mathematics, vol. 108, pp. 109-138, 1998. 


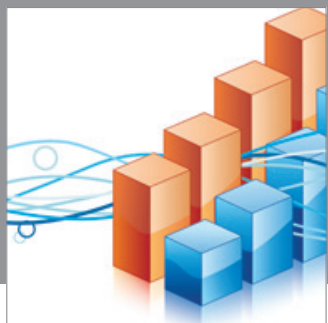

Advances in

Operations Research

mansans

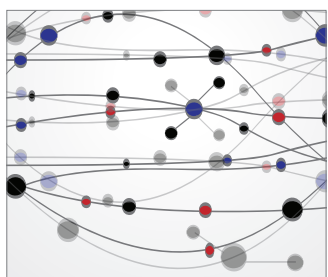

The Scientific World Journal
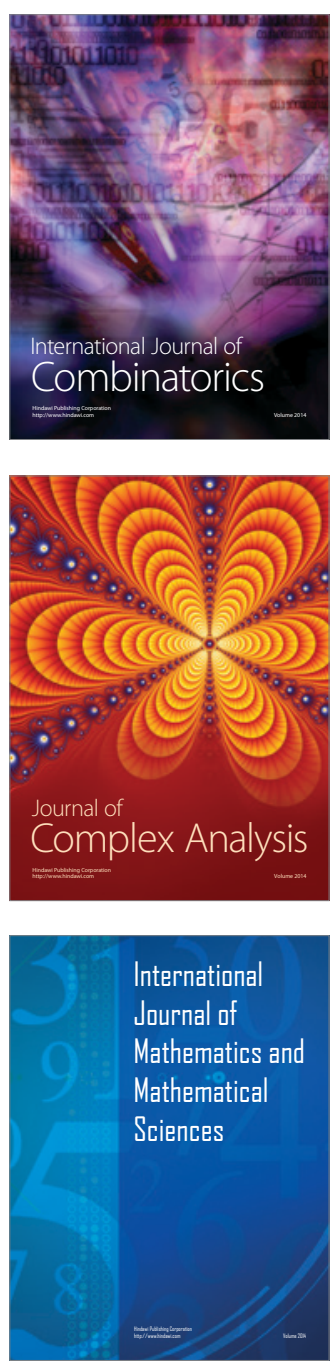
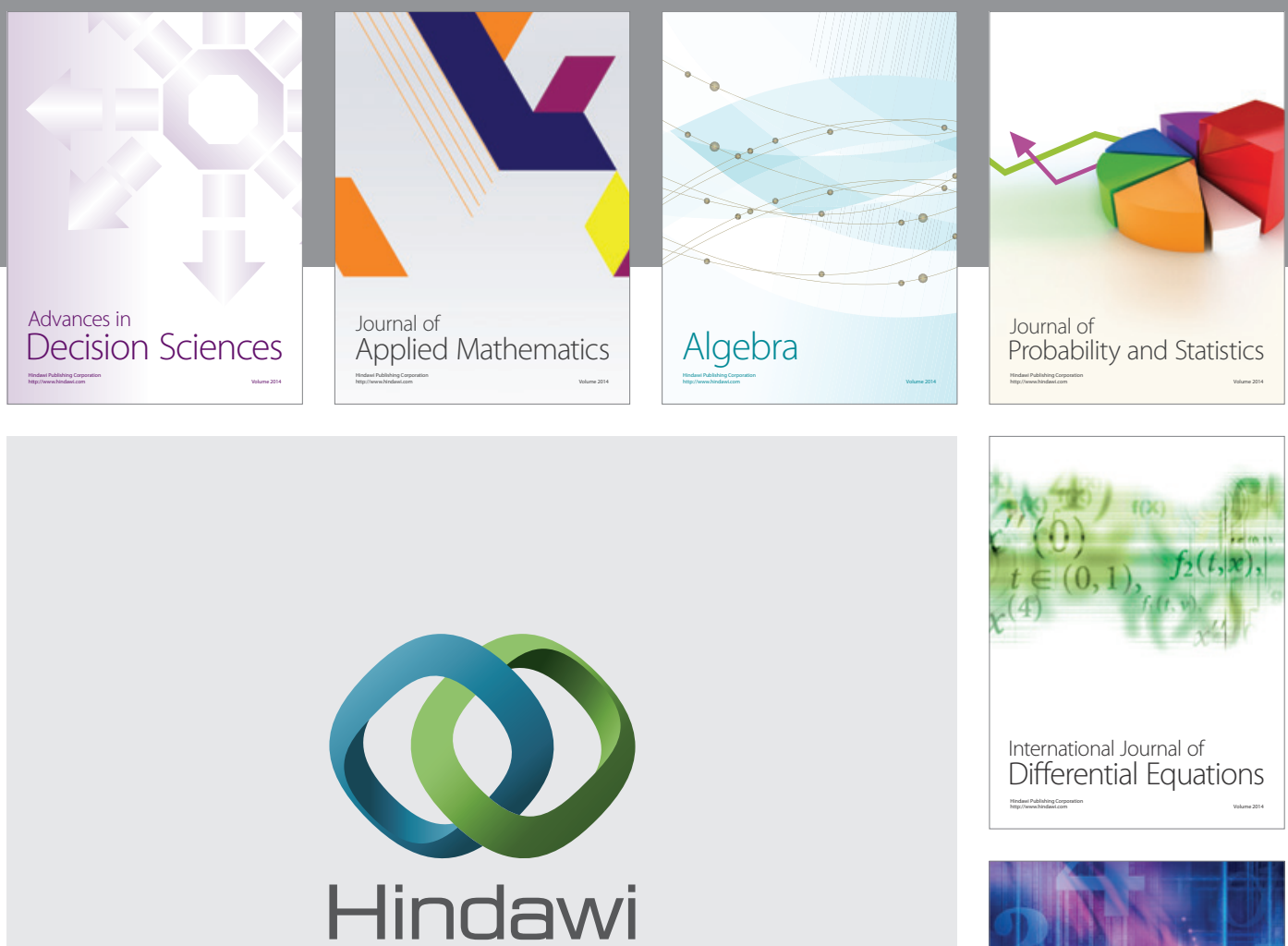

Submit your manuscripts at http://www.hindawi.com
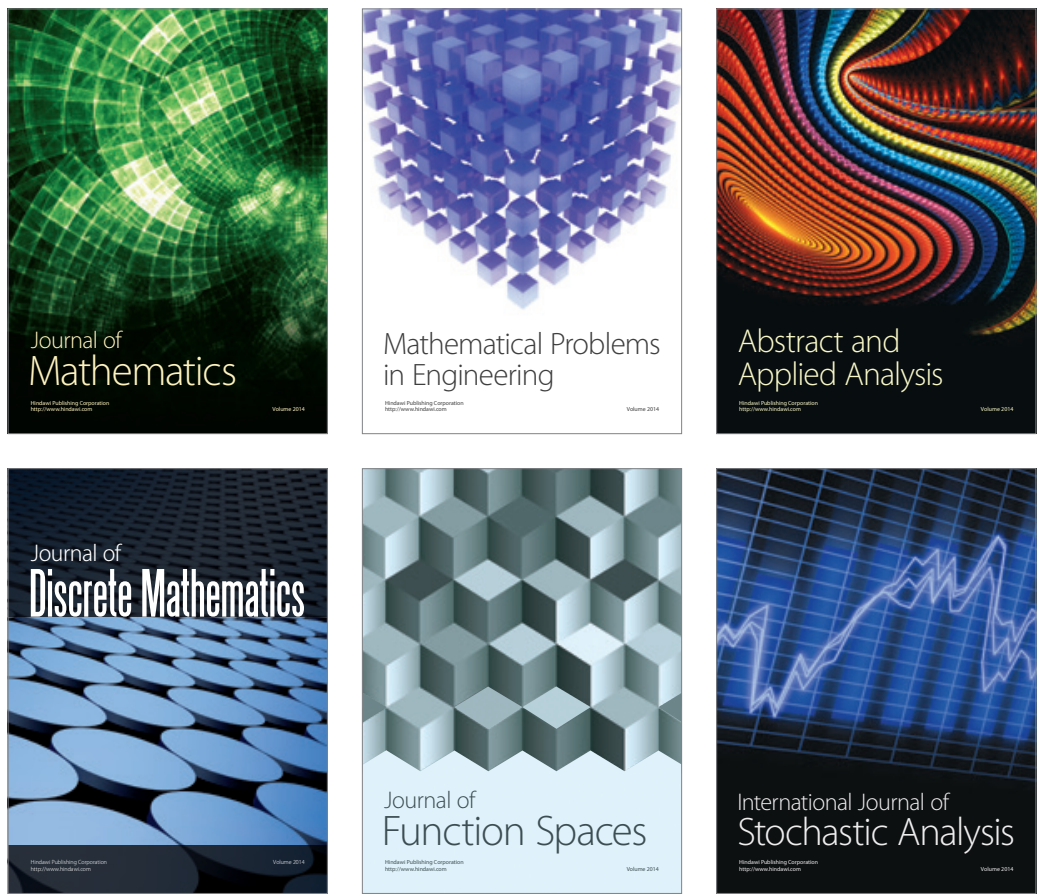

Journal of

Function Spaces

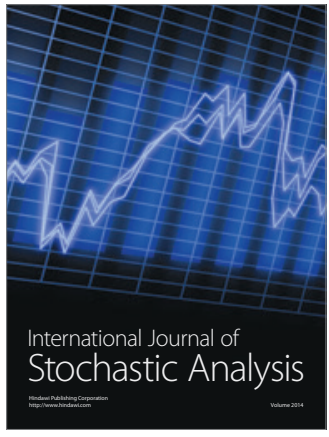

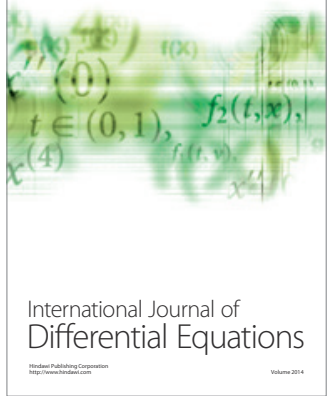
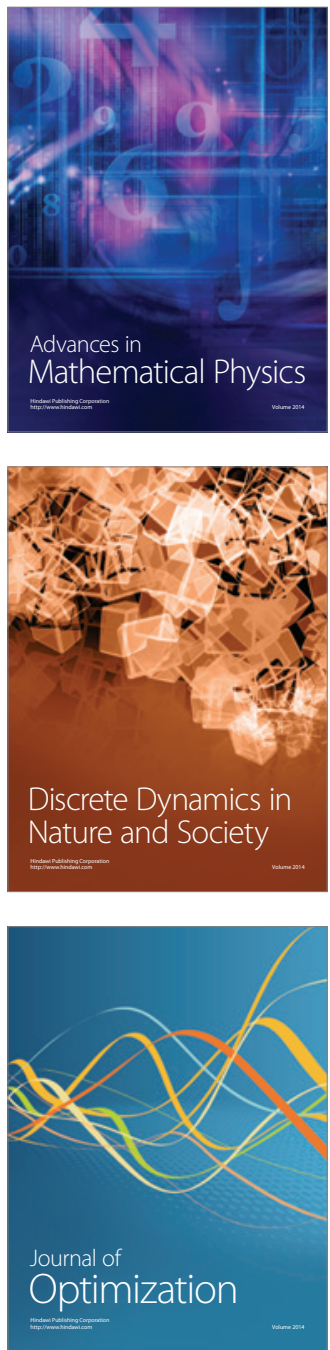